\title{
Surface charge of supramolecular nanosystems for in vivo biodistribution: a microSPECT/CT imaging study
}

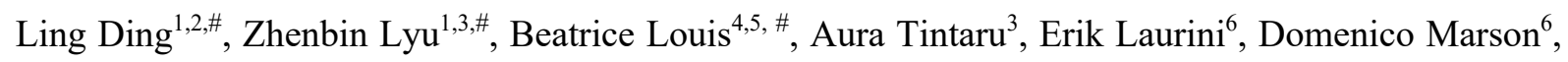
Mengjie Zhang ${ }^{7}$, Wanxuan Shao ${ }^{7}$, Yifan Jiang ${ }^{1}$, Ahlem Bouhlel ${ }^{4,5}$, Laure Balasse ${ }^{4,5}$, Philippe Garrigue ${ }^{4,5}$, Eric Mas ${ }^{8}$, Suzanne Giorgio ${ }^{1}$, Juan Iovanna ${ }^{8}$, Yuanyu Huang ${ }^{7}$, Sabrina Pricl ${ }^{6,9}$, Benjamin Guillet $^{4,5}$, Ling Peng ${ }^{1, *}$

${ }^{1}$ Aix Marseille Univ, CNRS, Centre Interdisciplinaire de Nanoscience de Marseille (CINaM), UMR 7325, Equipe Labellisée Ligue Contre le Cancer, Marseille, France

${ }^{2}$ Aix-Marseille Univ, CNRS, Centre de Résonance Magnétique Biologique et Médicale (CRMBM), UMR 7339, Marseille, France

${ }^{3}$ Aix Marseille Univ, CNRS, Institut de Chimie Radicalaire (ICR), Marseille, France

${ }^{4}$ Aix Marseille Univ, INSERM, INRAE, C2VN, Marseille, France

${ }^{5}$ Aix Marseille Univ, CNRS, CERIMED, Marseille, France

${ }^{6}$ Molecular Biology and Nanotechnology Laboratory (MolBNL@UniTS), DEA, University of Trieste, Trieste, Italy

${ }^{7}$ School of Life Science; Advanced Research Institute of Multidisciplinary Science; Institute of Engineering Medicine; Key Laboratory of Molecular Medicine and Biotherapy; Beijing Institute of Technology, Beijing, China.

${ }^{8}$ Aix Marseille Univ, INSERM, CRCM, Marseille, France

${ }^{9}$ Department of General Biophysics, Faculty of Biology and Environmental Protection, University of Lodz, Lodz, Poland

${ }^{*} \mathrm{LD}, \mathrm{ZL}, \mathrm{BL}$ contributed equally

\section{*Corresponding author:}

Dr Ling Peng

Aix Marseille Univ, CNRS, Centre Interdisciplinaire de Nanoscience de Marseille (CINaM), Marseille, France

Email: ling.peng@univ-amu.fr

ORCID: 0000-0003-3990-5248 


\begin{abstract}
:
Bioimaging has revolutionized medicine by providing accurate information for disease diagnosis and treatment. Nanotechnology-based bioimaging is expected to further improve imaging sensitivity and specificity. In this context, we have developed supramolecular nanosystems based on self-assembly of amphiphilic dendrimers for single photon emission computed tomography (SPECT) bioimaging. These dendrimers bear multiple $\mathrm{In}^{3+}$ radionuclides at their terminals as SPECT reporters. By replacing the macrocyclic DOTA cage with the smaller NOTA scaffold as the $\mathrm{In}^{3+}$ chelator, the corresponding dendrimer exhibited neutral $\mathrm{In}^{3+}$ complex terminals in place of negatively charged $\mathrm{In}^{3+}$-complex terminals. This negative-toneutral surface charge alteration completely reversed the zeta-potential of the nanosystems from negative to positive. As a consequence, the resulting SPECT nanoprobe generated a highly sought-after biodistribution profile accompanied by a drastically reduced uptake in liver, leading to significantly improved tumor imaging. This finding contrasts with current literature reporting that positively charged nanoparticles have preferential accumulation in the liver. As such, this study provides new perspectives for improving the biodistribution of positively charged nanosystems for biomedical applications.
\end{abstract}

\title{
Keywords:
}

supramolecular nanosystems, dendrimer, self-assembling, surface charge, metal chelators, SPECT imaging 


\section{Introduction}

Molecular imaging has revolutionized cancer management by providing precise information relating to tumor detection, grading, staging, and diagnosis, as well as monitoring treatment response and efficacy for personalized medicine. ${ }^{[1,2]}$ Nevertheless, there is high demand for further improvements in terms of sensitivity, specificity, and spatial resolution. Nanotechnology is expected to overcome these limitations by further improving imaging sensitivity and specificity via the so-called 'enhanced permeability and retention (EPR)' effect, also termed passive tumor targeting. ${ }^{[3-5]}$ EPR allows nanosized macromolecules or particles to preferentially accumulate in tumor tissue because of the leaky vasculature and disabled lymphatic system characterizing the tumor microenvironment. ${ }^{[6]}$ As a result, the local concentration of imaging agents in tumor lesions can be significantly increased, leading to better imaging outcomes. Moreover, nanosystems can carry multiple or hundreds of imaging reporters, which can significantly enhance the contrast signal for more accurate imaging and diagnosis. Consequently, different nanosystems have been explored and studied for tumor imaging. ${ }^{[3-5]}$

Dendrimer nanosystems are of particular interest for the delivery of imaging agents because of the unique dendritic structure and multivalent cooperativity confined within the nanoscale dimension. ${ }^{[7-12]}$ We have recently established small amphiphilic dendrimers that are able to self-assemble into supramolecular nanosystems for effective tumor imaging using positron emission tomography (PET) and single photon emission computed tomography (SPECT) (Figure 1). ${ }^{[13,14]}$ Both PET and SPECT are radio-imaging techniques that have a high sensitivity yet unlimited tissue penetration, and are able to visualize functional information quantitatively. ${ }^{[15,16]}$ Notably, SPECT is the most prevalent clinical imaging modality, accounting for $>75 \%$ of all nuclear imaging procedures. ${ }^{[17]}$ Thus, we have focused our recent efforts on optimizing our supramolecular dendrimer systems for SPECT imaging. 
We previously developed an amphiphilic dendrimer In-1 composed of a long hydrophobic alkyl chain and a hydrophilic poly(amidoamine) dendron bearing the SPECT radionuclide ${ }^{111} \operatorname{In}(\mathrm{III})$ complexed with the macrocycle DOTA $(1,4,7,10-$ tetraazacyclododecane-1,4,7,10-tetraacetic acid) ring in the chelator DOTAGA $(1,4,7,10$ tetraazacyclododececane-1-(glutaric acid)-4,7,10-triacetic acid) at the terminals (In-1 in Figure 1). ${ }^{[14]}$ By virtue of its amphiphilic nature, In-1 self-assembled into small and stable supramolecular nanomicelles for effective SPECT imaging of tumors based on the favorable combination of EPR-based passive tumor targeting and the dendrimeric structure bearing multivalent SPECT reporters. Nevertheless, In-1 also displayed high liver retention, which represents a severe limitation for its future clinical translation.

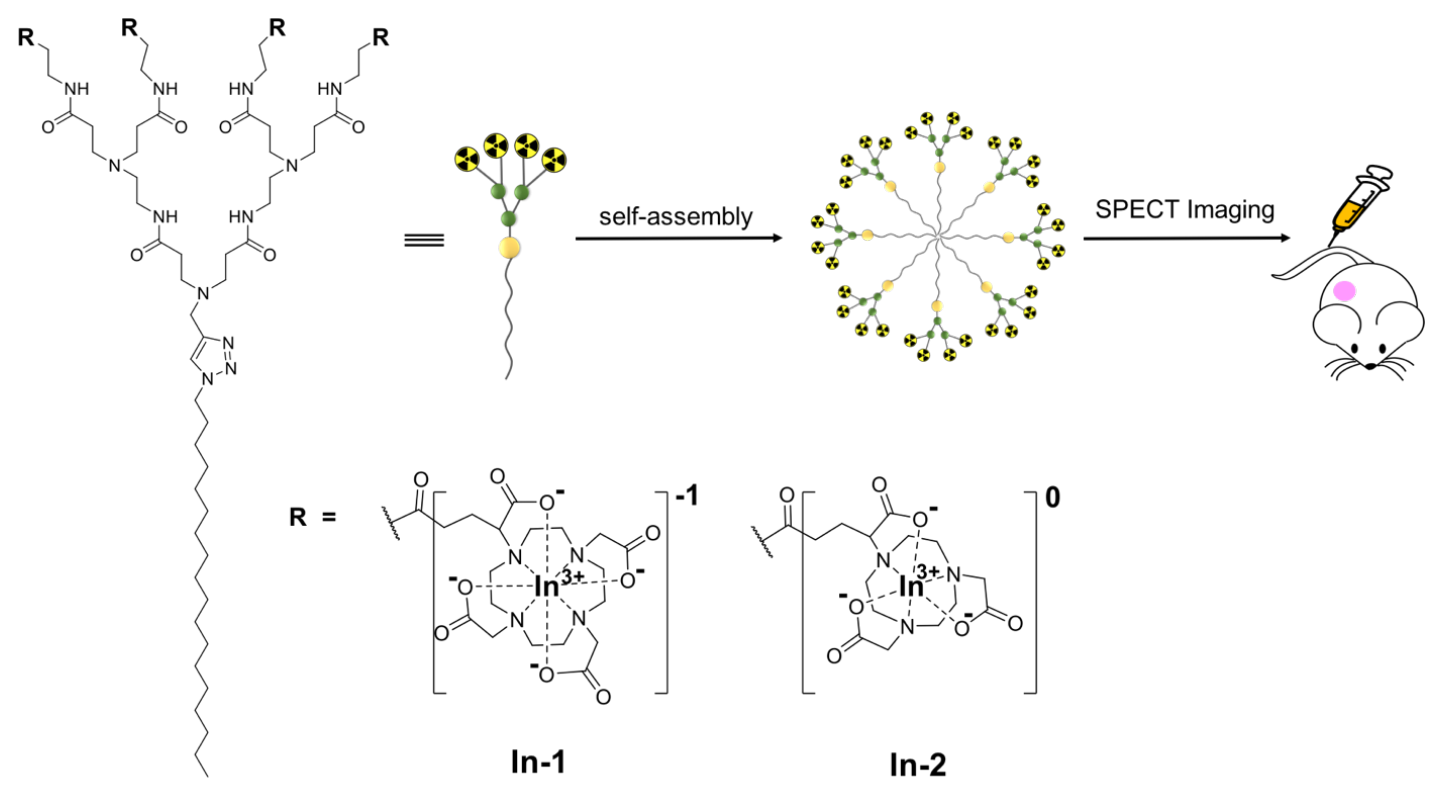

Figure 1. Schematic illustration of the supramolecular dendrimer nanosystems, based on the self-assembling amphiphilic dendrimers $\mathbf{I n - 1}$ and $\mathbf{I n - 2}$ bearing radionuclide $\mathrm{In}^{3+}$ terminals complexes with the macrocyclic DOTA and NOTA cages in the DOTAGA and NODAGA chelators, respectively, for single photon emission computed tomography (SPECT) imaging of tumors. 
It is well known that different chelators can significantly impact the biodistribution of radiotracers based on their size, charge, geometry, and lipophilicity when complexed with radionuclide metal ions. ${ }^{[18-20]}$ As the macrocycle DOTA ring in the DOTAGA chelator forms a negatively charged complex with $\mathrm{In}^{3+}$, we suspected that, despite the small nanosize of the corresponding In-1 nanomicelle, the overall surface charge of the In-1 nanoparticle might cause the high liver uptake and retention. Thus, we hypothesized that suppressing the negative surface charge of the dendrimer could limit this unfavorable liver retention. Accordingly, we replaced the macrocyclic DOTA cage with the NOTA $(1,4,7-$ triazacyclononane-1,4,7-triacetic acid) scaffold by conjugating the chelator NODAGA (1,4,7-triazacyclononane,1-glutaric acid-4,7-acetic acid) at the dendrimer terminals, because the NOTA ring can form a neutral complex when chelating the trivalent metal ion $\operatorname{In}^{3+}(\mathbf{I n}-$ 2 in Figure 1). ${ }^{[21]}$ Also, the macrocycle NOTA scaffold is smaller than the DOTA cage, and hence generates more stable complexes with small metal ions such as $\mathrm{Ga}^{3+}, \mathrm{In}^{3+}$, and $\mathrm{Cu}^{2+} \cdot{ }^{[22]}$ It should be noted that here we use DOTA and NOTA cages or rings as macrocyclic scaffolds for complexing with metal ions, rather than as the specific chelators in this work. Their corresponding chelators used in this work, DOTAGA and NODAGA, are, respectively, DOTA and NOTA-derivatives, often used for convenient conjugation with other chemical entities to present DOTA and NOTA scaffolds, thus maintaining the full denticity of DOTA and NOTA when chelating with metal ions.

Indeed, changing the DOTA cage to the NOTA ring at the amphiphilic dendrimer terminals had a profound impact on the surface charge of the resulting dendrimer, completely reversing the zeta potential from negative to positive when complexing with the trivalent metal ion $\mathrm{In}^{3+}$. As a consequence of this alteration, the radioactive $\mathrm{In}^{3+}$-labelled imaging nanoprobe In-2 led to a highly favorable biodistribution with drastically reduced uptake in liver, generating 
significantly improved tumor imaging. Although current literature reports that positively charged nanoparticles preferentially accumulate in the liver, ${ }^{[3-5]}$ our results clearly show that the presence of neutral surface regions of nanosystem with overall positively charged zeta potential could also result in drastically reduced liver uptake. Therefore, our findings provide new perspectives for improving the safety and biodistribution of various nanosystems for biomedical applications. Herein, we present and discuss our work in establishing In-2 as a promising agent for SPECT imaging of tumors, highlighting the importance of surface charge for the biodistribution of nanoparticles as a general concept.

\section{Results and discussion}

We prepared the dendrimer 2 bearing the macrocyclic NOTA cages at the terminals according to our previously reported synthesis. ${ }^{[13]}$ However, in the present study, we reduced the quantity of the reagent NODA-GA $(t \mathrm{Bu})_{3}$ by half in order to facilitate the purification procedures while maintaining the chemical integrity and high yield of product 2 (Figure 2A and

Scheme S1). Chelation of the stable isotope ${ }^{115} \mathrm{In}^{3+}$ by 2 was performed using ${ }^{115} \mathrm{InCl}_{3}$ at $25^{\circ} \mathrm{C}$ for $10 \mathrm{~min}$ at $\mathrm{pH} 4.0-4.5$ (Figure $2 \mathrm{~A}$ ). These conditions contrasted with those used for the synthesis of In-1, which required substantially higher temperatures $\left(55^{\circ} \mathrm{C}\right)$ and longer times (120 min). The successful complexation of four ${ }^{115} \mathrm{In}^{3+}$ ions by the macrocyclic NOTA scaffolds in 2 was confirmed using high-resolution mass spectroscopy, which revealed the isotopic pattern characteristic of the triply charged species $\left[{ }^{115} \mathbf{I n}-\mathbf{2}+3 \mathrm{H}\right]^{3+}$ of the expected molecular structure (Figure 2C, Figure S1). ${ }^{[23]}$ 


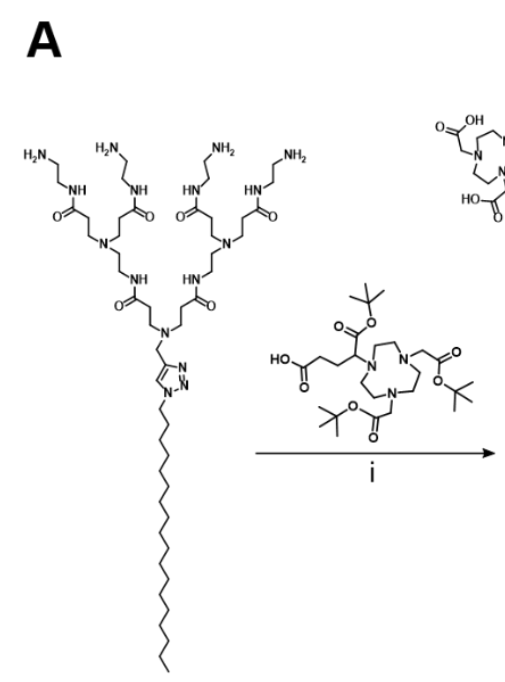

B

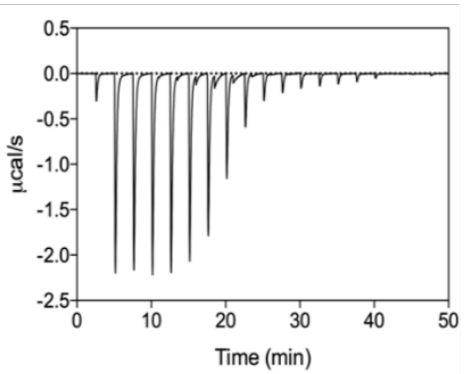

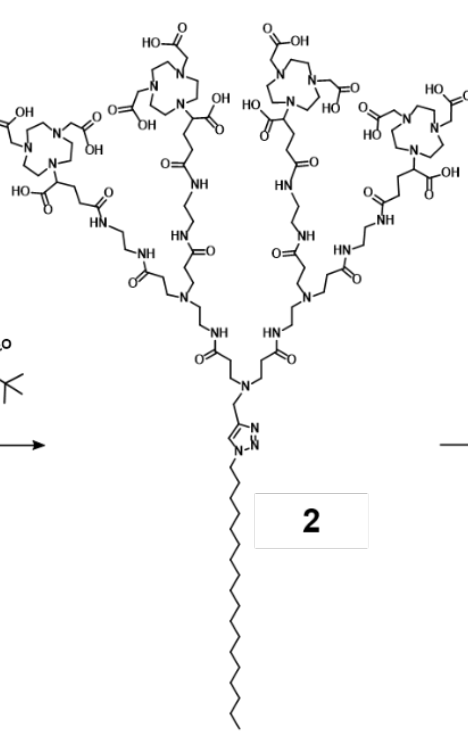

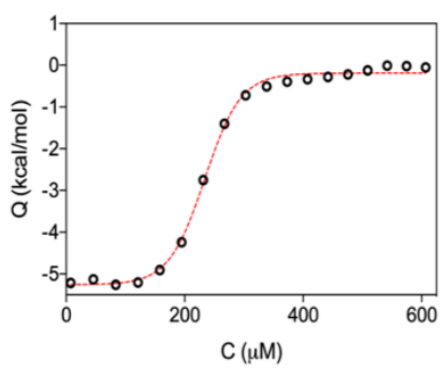

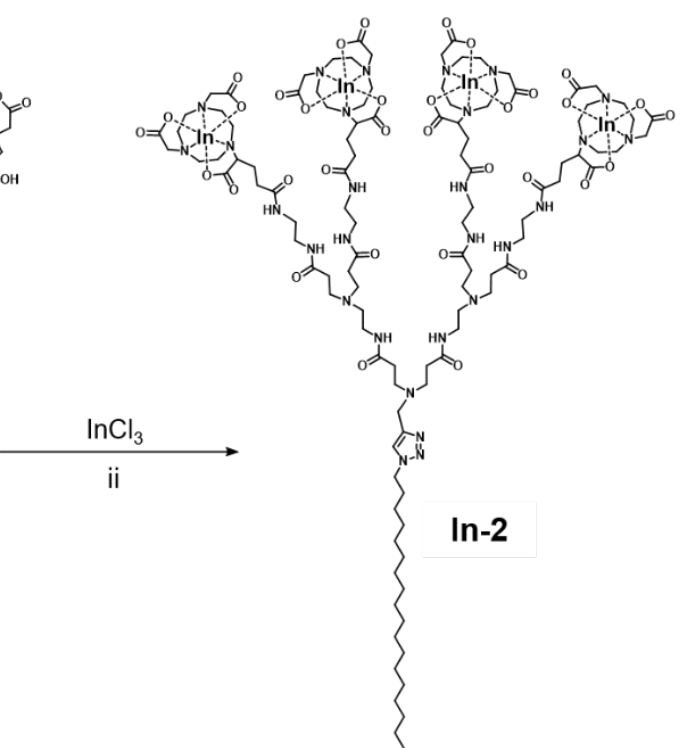

C

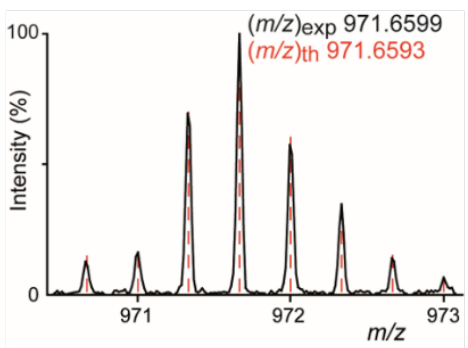

Figure 2. Synthesis of the amphiphilic dendrimer $\mathbf{2}$ and its chelation with the nonradioactive isotope $\left[{ }^{115} \mathrm{In}\right] \mathrm{In}^{3+}$ at the terminals. (A) Synthesis scheme: (i) (a) NODA-GA( $\left.t \mathrm{Bu}\right)_{3}, \operatorname{PyBOP}$, $\mathrm{NMM}, \mathrm{DMF}, 30^{\circ} \mathrm{C}, 72 \mathrm{~h}$; (b) TFA, $\mathrm{CH}_{2} \mathrm{Cl}_{2}, 30^{\circ} \mathrm{C}, 16$ h. (ii) $\left[{ }^{115} \mathrm{In}\right] \mathrm{InCl}_{3}, 1.0 \mathrm{M} \mathrm{HCl}, 24^{\circ} \mathrm{C}, 10$ min. (B) Isothermal titration calorimetry curve (right) for chelation of $\operatorname{In}^{3+}$ with the dendrimer 2. The left panel shows measured heat power versus time elapsed during titration. (C) Highresolution mass spectrum showing the isotopic pattern characteristic of the triply charged species $\left[\left[{ }^{115} \mathrm{In}\right] \mathbf{I n}-\mathbf{2}+3 \mathrm{H}\right]^{3+}$, which overlaps with the theoretical value presented with the red dashed line.

Previously, we also supported the synthesis of the dendrimer In-1 carrying the DOTA cages through isothermal titration calorimetry (ITC). ${ }^{[14]}$ We used the same approach to study the thermodynamics of the interaction between $\mathrm{In}^{3+}$ and the dendrimer 2 bearing the NOTA 
cages for generating In-2 (Figure 2B). The titration of both dendrimers $\mathbf{1}$ and $\mathbf{2}$ with the trivalent cation $\mathrm{In}^{3+}$ presented very similar calorimetric behaviors. The spontaneous formation of In-2 was promoted by a favorable binding free energy $(\Delta \mathrm{G})$ of $-7.64 \mathrm{kcal} \mathrm{mol}^{-1}$. This value arises from the balanced and favorable contributions of both the entropic $\left(-\mathrm{T} \Delta \mathrm{S}=-2.21 \mathrm{kcal} \mathrm{mol}^{-1}\right)$ and the enthalpic $\left(\Delta \mathrm{H}=-5.43 \mathrm{kcal} \mathrm{mol}^{-1}\right)$ components, which are similar to the binding thermodynamic parameters for In-1 $\left(\Delta \mathrm{G}=-7.86 \mathrm{kcal} \mathrm{mol}^{-1},-\mathrm{T} \Delta \mathrm{S}=-2.61\right.$, and $\Delta \mathrm{H}=-5.25$ kcal $\mathrm{mol}^{-1}$ ). A 4:1 stoichiometry was also determined for the In-2 complex by the ITC-derived number of occupied sites $(n=3.95)$, corroborating the results obtained using high-resolution mass spectroscopy (Figure 2C).

With the synthesized In-2 in hand, we then examined the spontaneously self-assembling features of In-2 in water. Using transmission electron microscopy, we observed the formation of small and spherical nanoparticles of an average size of $18 \mathrm{~nm}$ by $\mathbf{I n - 2}$ in water (Figure 3A), similar to those generated by the self-assembly of In-1 (Figure 3F). Furthermore, dynamic light scattering analysis confirmed the presence of nanoparticles of similar sizes for both dendrimers In-1 and In-2 in water and in phosphate buffer at pH 7.4 (Figure 3B, G, and Figure S3). This highlighted that, like In-1, In-2 also self-assembled into nanomicelles. We then used a fluorescent spectroscopic assay with Nile Red to estimate the critical micelle concentration (CMC) of In-2, which was around $40 \mu \mathrm{M}$, similar to the CMC value for In-1 (49 $\mu \mathrm{M})$ (Figure S4). In addition, we measured the surface zeta potential of the In-2 nanomicelles, and obtained a positive value of $+11 \mathrm{mV}$ (Figure $3 \mathrm{C}$ ), which could be ascribed to the interior tertiary amine functionality, since the $\mathrm{In}^{3+}$ complex with the NOTA ring in the NODAGA chelator at the dendrimer terminal is neutral. This is distinctly different from that of In-1, where each DOTA cage within the DOTAGA terminal in complex with $\operatorname{In}^{3+}$ has a net negative charge, thereby generating In-1 nanomicelles with an overall negative zeta potential of $-8 \mathrm{mV}$ (Figure $3 \mathrm{H}$ ). 
A

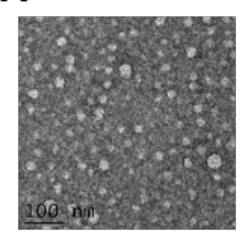

$\mathbf{F}$

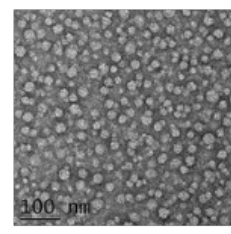

B

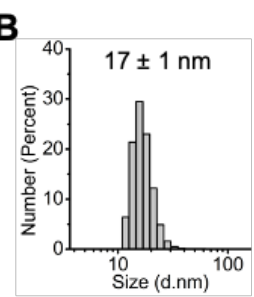

G

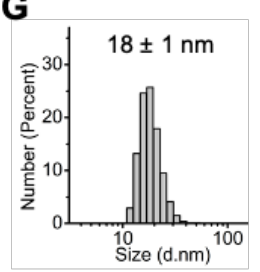

C

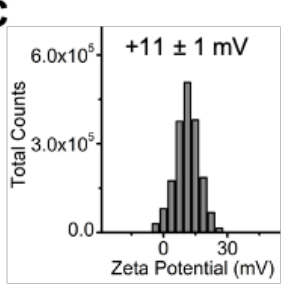

H

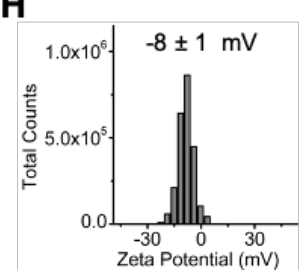

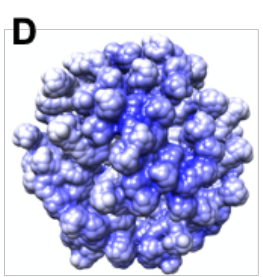

E
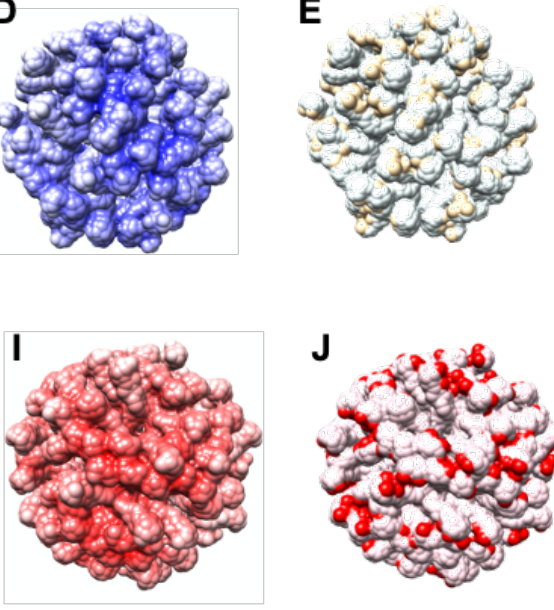

$\mathbf{J}$

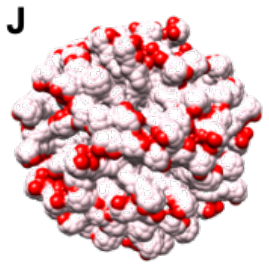

Figure 3. Comparison of the spontaneous self-assembling of the amphiphilic dendrimers In-1 and In-2 into small and uniform nanomicelles in water. (A, F) Transmission electron microscopic imaging, $(B, G)$ dynamic light scattering analysis, $(C, H)$ surface zeta-potential measured using a Zeta-nanosizer, (D, I) electrostatic surface potential of the self-assembled nanostructures as extracted from the corresponding equilibrated molecular dynamics simulations, and (E, J) representation of the surface charge distribution localized on the $\mathrm{In}^{3+} /$ NOTA complexes at the NODAGA terminals (neutral, ivory) and the $\operatorname{~}^{3+} /$ DOTA at the DOTAGA terminals (negative, red) for dendrimers In-2 (upper row) and In-1 (lower row). In panels D and I, the red color represents a negatively charged surface, the dark blue color represents a positively charged surface, while the white color represents a neutral surface.

To further confirm the spontaneous self-assembly of In-1 and In-2 into nanomicelles, we performed molecular dynamics simulations following a consolidated procedure. ${ }^{[13,14]}$ Starting from randomly distributed monomers, we obtained stable spherical micelles for both systems during the timescale of the simulations $(1.0 \mu \mathrm{s})$ (Figures 3D, F, I, J). The corresponding average micelle diameters computed from the equilibrated molecular dynamics simulations were $15 \mathrm{~nm}$ and $13 \mathrm{~nm}$ for the In-1 and In-2 systems, respectively, which were in good agreement with the experimental results obtained using both dynamic light scattering and transmission electron 
microscopy. By inspecting the conformational structures of both micelles, no back-folding of the terminal groups was detected, in line with our previous findings obtained for similar systems $[13,14]$. Accordingly, the $\mathrm{In}^{3+}$-bearing terminal units are all located at the periphery of the micelles in both the In-1 and In-2 systems. Further analysis of the electrostatic surface potential confirmed the foreseen effect of replacing the DOTA cage with the NOTA scaffold, with a negative electrostatic potential observed for In-1 (Figure 3I) and a positive potential for In-2 (Figure 3D), in agreement with the experimentally-determined surface zeta potentials (Figures 3C, H). Moreover, the surface of the In-1 micelle was characterized by the presence of localized negatively charged regions, corresponding to the trivalent $\operatorname{In}^{3+}$ ions in complex with the DOTA cages at the DOTAGA terminals (Figure 3J), while the In-2 nanoparticles presented patches of neutral charge, corresponding to $\mathrm{In}^{3+}$ ions in complex with NOTA rings at the NODAGA terminals (Figure 3E).

Next, we prepared the radioactive dendrimer complex $\left[{ }^{111} \mathrm{In}\right] \mathbf{I n}-\mathbf{2}$ for SPECT imaging. Radiolabeling was performed using $\left[{ }^{111} \mathrm{In}\right] \mathrm{In}^{3+}$ in ammonium acetate buffer, and the obtained $\left[{ }^{111} \mathrm{In}\right] \mathbf{I n}-2$ complex had an excellent radiochemical purity of over 93\%, which was remarkably stable and maintained up for at least $48 \mathrm{~h}$ at $37^{\circ} \mathrm{C}$ in human serum (Figure 4A). SPECT imaging using $\left[{ }^{111} \mathrm{In}\right] \mathbf{I n}-2$ was first performed in orthotopically xenografted mice bearing human pancreatic adenocarcinoma SOJ-6 tumors, and the results obtained with $\left[{ }^{111} \mathrm{In}\right] \mathbf{I n}-\mathbf{1}$ were used as control for comparison. Co-registration with computed tomography (CT) enabled anatomical localization of the SPECT signals for further quantification. As seen in Figure 4B, a significantly improved image contrast for tumor visualization was obtained with $\left[{ }^{111} \mathrm{In}\right] \mathbf{I n}-\mathbf{2}$ compared to $\left[{ }^{111} \operatorname{In}\right] \mathbf{I n}-\mathbf{1}$. 

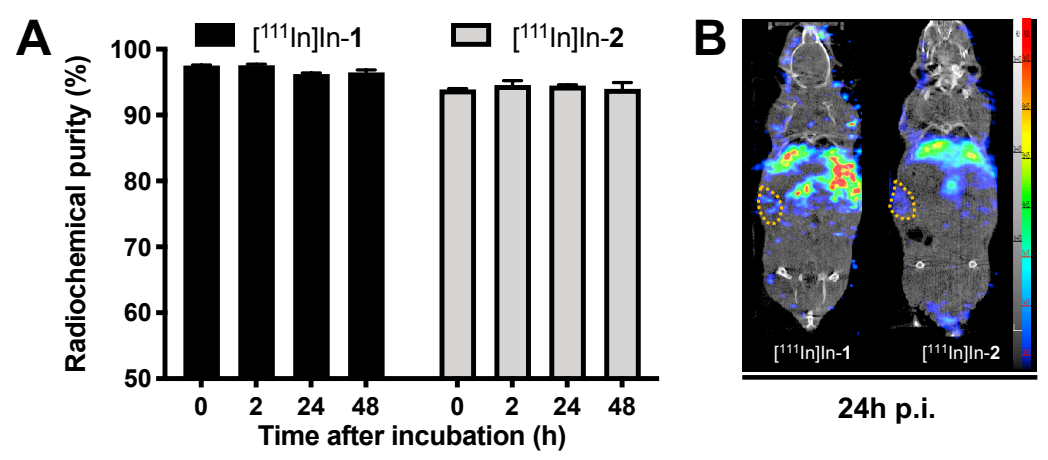

Figure 4. Radiolabeled dendrimers $\left[{ }^{111} \mathrm{In}\right] \mathbf{I n}-\mathbf{1}$ and $\left[{ }^{111} \mathrm{In}\right] \mathbf{I n}-\mathbf{2}$ for SPECT imaging in a mouse orthotopic xenograft model of pancreatic adenocarcinoma (SOJ-6 cell line) $24 \mathrm{~h}$ post-injection (p.i.). (A) Radiochemical purity and stability of [ $\left[{ }^{111} \mathrm{In}\right] \mathbf{I n}-\mathbf{1}$ and $\left[{ }^{111} \mathrm{In}\right] \mathbf{I n}-\mathbf{2}$ assessed by instant thin layer chromatography immediately after incubation with human serum at $37^{\circ} \mathrm{C}$, and after $2 \mathrm{~h}, 24 \mathrm{~h}$ and $48 \mathrm{~h}$, respectively. Results show excellent radiochemical purities up to $48 \mathrm{~h}$ after radiosynthesis. (B) Representative $\mu \mathrm{SPECT} / \mathrm{CT}$ maximum intensity projection images of $\left[{ }^{111} \mathrm{In}\right] \mathbf{I n}-\mathbf{1}$ (left) and $\left[{ }^{111} \mathrm{In}\right] \mathbf{I n}-2$ (right) $24 \mathrm{~h}$ after intravenous injection. The tumor is highlighted by orange dashed circles.

The improved tumor imaging achieved with $\left[{ }^{111} \mathrm{In}\right] \mathbf{I n}-\mathbf{2}$ compared to $\left[{ }^{111} \mathrm{In}\right] \mathbf{I n}-\mathbf{1}$ was further confirmed using a patient-derived xenograft model of pancreatic cancer (L-IPC cell line). As illustrated in Figure 5A, 5B, and 5C, $\left[{ }^{111} \mathrm{In}\right] \mathbf{I n}-\mathbf{2} \mu$ SPECT signal quantification in the liver was significantly reduced compared to that of $\left[{ }^{111} \mathrm{In}\right] \mathbf{I n}-\mathbf{1}$ as soon as $2 \mathrm{~h}$ post-injection, and was maintained even $24 \mathrm{~h}$ and $48 \mathrm{~h}$ post-injection. Notably, the liver uptake was reduce by more than two times with $\left[{ }^{111} \operatorname{In}\right] \mathbf{I n}-2$ compared with $\left[{ }^{111} \operatorname{In}\right] \mathbf{I n}-1$. Meanwhile, in the kidneys, $\left[{ }^{111} \mathrm{In}\right] \mathbf{I n}-2 \mu$ SPECT signal quantification was elevated up to $20 \%$ versus that of $\left[{ }^{111} \mathrm{In}\right] \mathbf{I n}-\mathbf{1}$. Remarkably, the $\mu$ SPECT signal of both $\left[{ }^{111} \mathrm{In}\right] \mathbf{I n}-\mathbf{1}$ and $\left[{ }^{111} \mathrm{In}\right] \mathbf{I n}-\mathbf{2}$ was drastically reduced in organs likely to generate background such as the heart, lungs, brain, muscle, and bladder at 24 $\mathrm{h}$ and $48 \mathrm{~h}$ after administration, compared to quantifications performed $2 \mathrm{~h}$ post-injection, 
giving rise to better tumor imaging quality. When expressed as tumor-to-muscle or tumor-toliver ratios, $\mu \mathrm{SPECT} / \mathrm{CT}$ signal quantifications of tumor uptake of $\left[{ }^{111} \mathrm{In}\right] \mathbf{I n}-\mathbf{2}$ were significantly higher than those of $\left[{ }^{111} \mathrm{In}\right] \mathbf{I n}-1$, with up to a 2-fold increase (Figures 5D and 5E), translating into notably better tumor imaging quality.

A
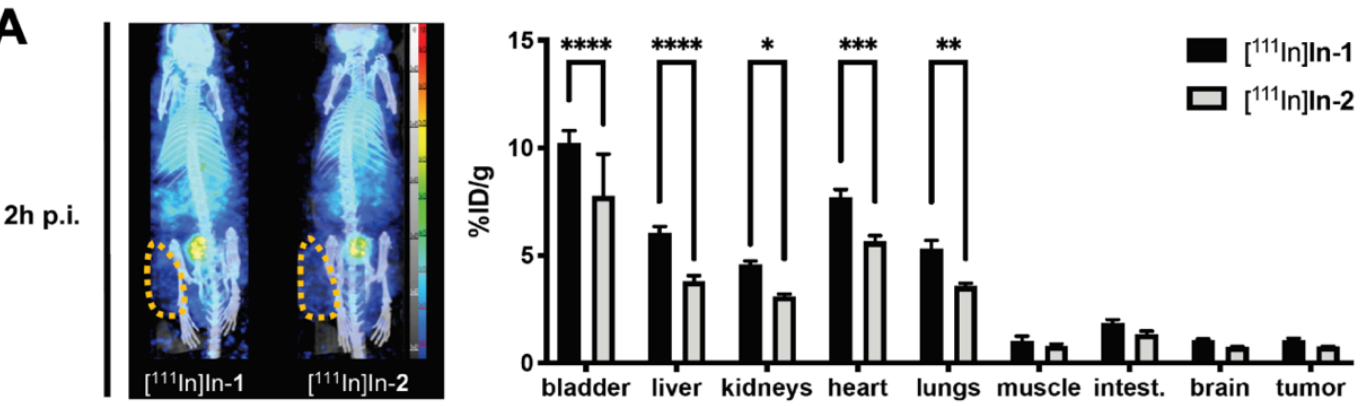

B
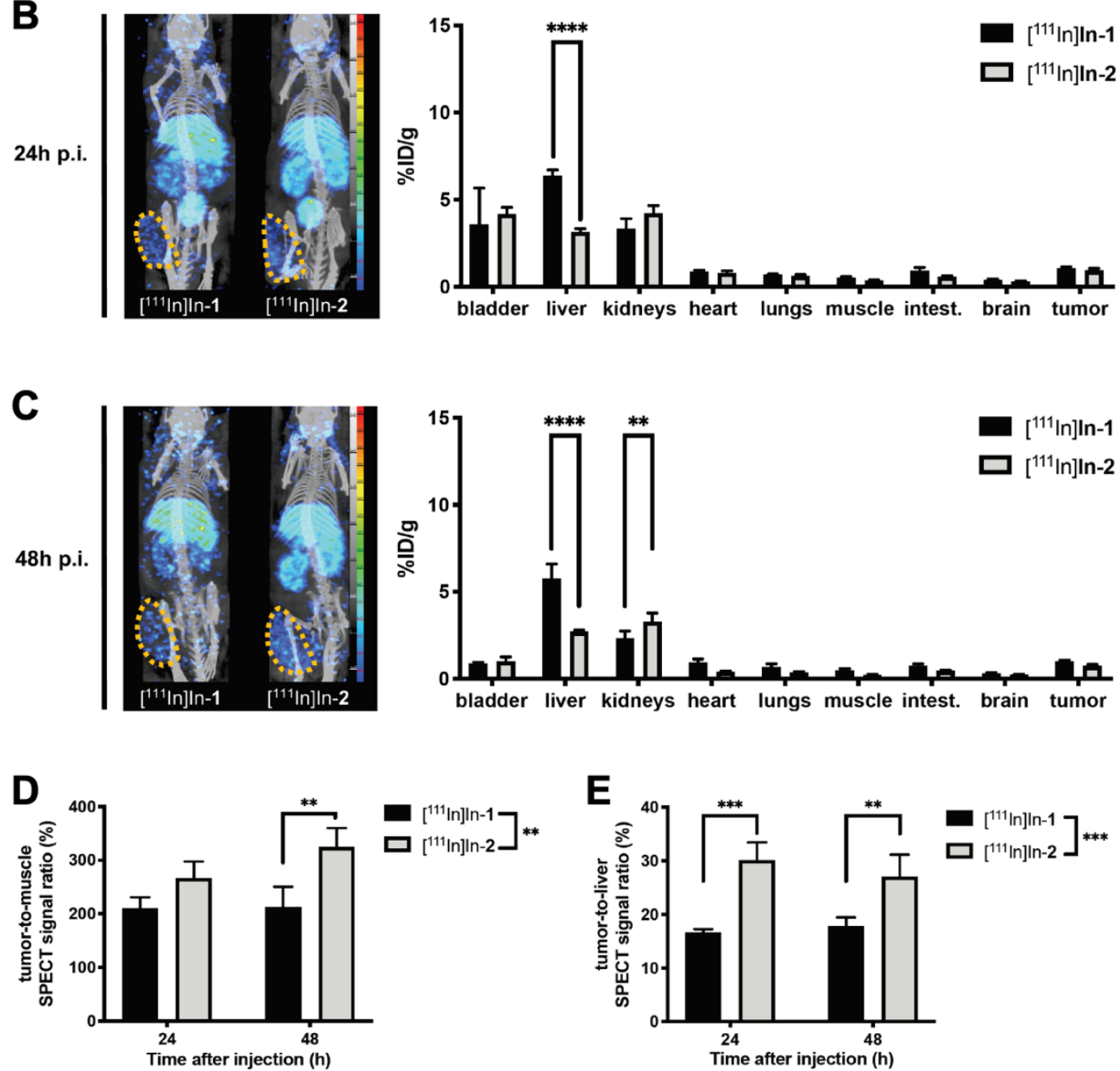
Figure 5. Radiolabeled dendrimer $\left[{ }^{111} \mathrm{In}\right] \mathbf{I n}-\mathbf{1}$ and $\left[{ }^{111} \mathrm{In}\right] \mathbf{I n}-\mathbf{2}$ for SPECT imaging in a patientderived subcutaneous xenograft model of pancreatic cancer (L-IPC). (A,B,C) Representative $\mu \mathrm{SPECT} / \mathrm{CT}$ maximum intensity projection images of $\left[{ }^{111} \mathrm{In}\right] \mathbf{I n}-\mathbf{1}$ and $\left[{ }^{111} \mathrm{In}\right] \mathbf{I n}-\mathbf{2} 2 \mathrm{~h}$ (A, left), $24 \mathrm{~h}(\mathrm{~B}$, left $)$, and $48 \mathrm{~h}(\mathrm{C}$, left) after intravenous injection. The tumor is highlighted in orange dashed circles. Biodistributions of $\left[{ }^{111} \mathrm{In}\right] \mathbf{I n}-\mathbf{1}$ and $\left[{ }^{111} \mathrm{In}\right] \mathbf{I n}-\mathbf{2}$ were quantified in each organ by $\mu \mathrm{SPECT} / \mathrm{CT} 2 \mathrm{~h}$ (A, right), $24 \mathrm{~h}$ (B, right) and $48 \mathrm{~h}$ (C, right) post-injection (p.i.). Data are expressed as the mean percentage of whole-body activity per gram of tissue at the time of acquisition ( $n=3$ mice). The two nanosystems showed significantly different signal quantifications $2 \mathrm{~h}$ p.i. in the bladder $(* * * * P<0.0001)$, liver $(* * * * P<0.0001)$, kidneys $(* P=$ $0.0118)$, heart $(* * * P=0.0002)$ and lungs $(* * P=0.0024), 24$ h p.i. in the liver $(* * * * P<0.0001)$, and $48 \mathrm{~h}$ p.i. in the liver $(* * * * P<0.0001)$ and kidneys $(* * P<0.0014)$, comparing $\left[{ }^{111} \mathrm{In}\right] \mathbf{I n}-2$ with [ ${ }^{111}$ In] In-1 (2-way ANOVA followed by a Sidak's post-hoc test). (D) Tumor-to-muscle ratio $\mu$ SPECT/CT signal quantifications of tumor uptake of $\left[{ }^{111} \operatorname{In}\right] \mathbf{I n}-\mathbf{2}$ were significantly higher than those of $\left[{ }^{111} \operatorname{In}\right] \mathbf{I n}-148 \mathrm{~h}$ p.i. $\left({ }^{* *} P=0.0052\right)$ and most interestingly over time $(* * P$ $=0.0018,2$-way ANOVA followed by a Sidak's post-hoc test). (E) Tumor-to-liver ratios $\mu$ SPECT/CT signal quantifications of tumor uptake of $\left[{ }^{111} \mathrm{In}\right] \mathbf{I n}-\mathbf{2}$ were significantly higher than those of $\left[{ }^{111} \mathrm{In}\right] \mathbf{I n}-124 \mathrm{~h}$ p.i. $(* * * P<0.0007), 48$ h p.i. $(* * P=0.0075)$ and most interestingly over time $(* * * P=0.0001,2$-way ANOVA followed by a Sidak's post-hoc test).

In line with our original hypothesis, we tentatively rationalized the discrepancies in biodistribution to the difference in chelators and the resulting alteration in surface charge of In$\mathbf{1}$ and $\mathbf{I n - 2}$, since the size variation between the In-1 and In-2 nanomicelles was very small (Figure 3). As discussed above, after changing the DOTA cage in In-1 to the NOTA scaffold in In-2 at the dendrimer terminals, the zeta potential of the corresponding $\mathrm{In}^{3+}$-labelled nanomicelles formed by these dendrimers changed accordingly (Figures $3 \mathrm{E}$ and 3J), leading to 
a more than 2-fold reduced accumulation of $\left[{ }^{111} \mathrm{In}\right] \mathbf{I n}-\mathbf{2}$ in the liver. Of note, the majority of published studies report that positively-charged nanoparticles are more likely to accumulate in the liver than their negatively charged counterparts. ${ }^{[3-5]}$ Our results, however, demonstrate that overall positively charged nanosystems exhibiting neutral regions on their surface can also exhibit reduced liver uptake. As a consequence, a significantly improved biodistribution and imaging profile was obtained with $\left[{ }^{111} \mathrm{In}\right] \mathbf{I n}-\mathbf{2}$ compared to [ $\left.{ }^{111} \mathrm{In}\right] \mathbf{I n}-\mathbf{1}$. Our findings highlight that the impact on pharmacokinetics and biodistribution is dependent not only on the choice of chelator, ${ }^{[22,24]}$ but also on the nature of the whole imaging probes. This overall feature may impact the ability of probes to bind to proteins in body fluids, depending on the different surface charges, and hence impacting the overall biodistribution. ${ }^{[25]}$ In addition, lowering the liver uptake can also help to improve the safety and biodistribution profiles of the supramolecular nanosystems that have been developed for biomedical applications in general. Moreover, the dendrimer In-2 enabled better tumor imaging, which was significantly enhanced (up to 2.0fold) and long-lasting (up to $48 \mathrm{~h}$ after injection) for both-tumor-to-muscle and tumor-to-liver ratios (Figures 5C and 5D). This feature is particular interesting in developing imaging-guided internal radiotherapy.

It is noteworthy that the mice receiving the radioactive $\left[{ }^{111} \mathrm{In}\right] \mathbf{I n}-\mathbf{2}$ did not display any abnormal behaviors or adverse effects during all experimental imaging procedures. Furthermore, healthy mice that received the nonradioactive In-2 did not exhibit any organ damage or blood biochemistry defects, even when the administered dose of In-2 was 10 times higher than that required for SPECT imaging (Figure 6). As shown in Figure 6A, histological analysis of organs from mice treated with In-2 revealed no gross lesions or significant underlying pathologies in any organ tissue sections. Also, several major blood biochemistry parameters including alanine transaminase, aspartate transaminase, total bilirubin, creatinine, urea, total protein, alkaline phosphatase, triacylglycerol and total cholesterol, remained at the 
levels comparable to those found in untreated mice (Figure 6B). This highlighted that no acute events were associated with In-2 in terms of normal liver, kidney, and muscle function, further confirming that the main organs functioned well after treatment with In-2. All these data demonstrate that $\left[{ }^{111} \mathrm{In}\right] \mathbf{I n}-\mathbf{2}$ produces no adverse effects, but delivers effective SPECT imaging quality.
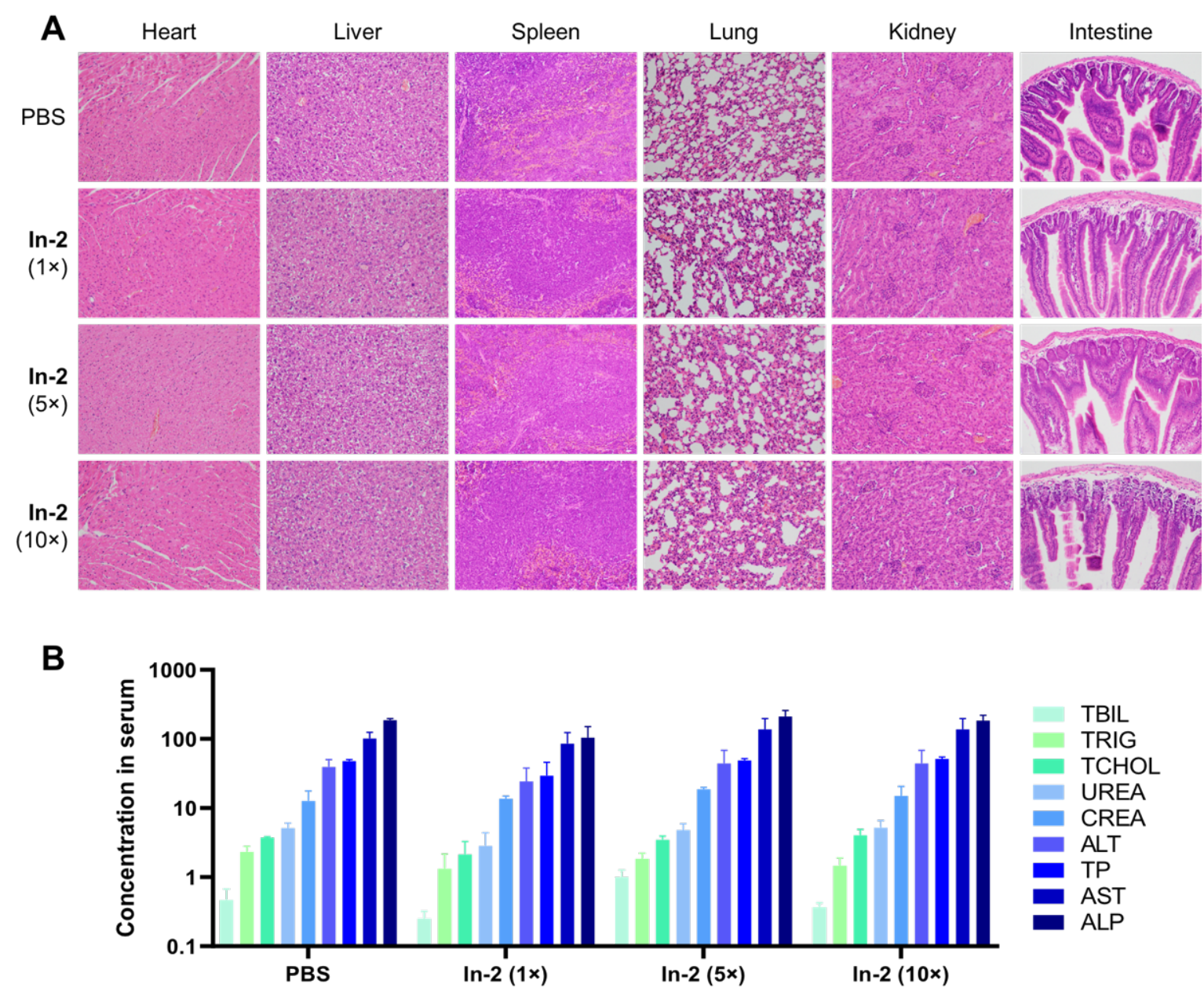

Figure 6. In vivo toxicity assessment of In-2 in healthy mice at different doses. $1 \times, 5 \times$, and $10 \times$ indicates that the formulations were administered at a dose equal to the SPECT-imaging dose, 5 times the imaging dose, and 10 times the imaging dose, respectively. (A) Histopathological analysis of the major organs from mice treated with In-2. Tissue samples were collected $24 \mathrm{~h}$ post-administration. No significant histopathological changes were observed in any of the tissue sections. Images were enlarged 200 times with the microscope. (B) Major serum biochemistry 
parameters measured in mouse serum collected at $24 \mathrm{~h}$ post-injection. Alanine transaminase (ALT), aspartate transaminase (AST), and alkaline phosphatase (ALP) were measured in U/L; urea, triacylglycerol (TRIG), and total cholesterol (TCHOL) were measured in $\mathrm{mmol} / \mathrm{L}$; creatinine (CREA) and total bilirubin (TBIL) were measured in $\mu \mathrm{mol} / \mathrm{L}$; total protein (TP) was measured in $\mathrm{g} / \mathrm{L}$. Data are shown as mean $\pm \mathrm{SD}$.

\section{Conclusion}

By replacing the DOTA cage with the NOTA scaffold to chelate the radionuclide $\mathrm{In}^{3+}$, we established a new amphiphilic dendrimer In-2 bearing neutral coordination complexes with the trivalent $\mathrm{In}^{3+}$ ions at the dendrimer terminals for SPECT imaging. This modification had a drastic impact, generating significantly improved tumor imaging and a beneficial biodistribution profile. Notably, the uptake of In-2 in the liver was reduced significantly, by more than 2-fold compared with that of In-1; at the same time, the imaging contrast was also considerably enhanced up to 2-fold and sustained even up to $48 \mathrm{~h}$ after injection. This study presents not only the supramolecular dendrimer nanosystem In-2 for safe and effective SPECT imaging of tumors, but also new perspectives for improving the safety and biodistribution of supramolecular nanosystems for biomedical applications such as theranostics based on imaging-guided internal radiotherapy.

\section{Acknowledgments}

We thank Michel Skandalovski (CERIMED, Aix-Marseille University), Samy Vigier, and Sandrine Pons (Faculty of Pharmacy, Aix-Marseille University) for technical support. This work was supported by the Ligue Nationale Contre le Cancer (LP, ZL), China Scholarship Council (LD), Italian Association for Cancer Research (IG17413) (SP), the French National 
Research Agency under the frame of the Era-Net EURONANOMED European Research projects 'NANOGLIO', 'TARBRAINFECT', and 'NAN-4-TUM' (LP), H2020 NMBP 'SAFEN-MEDTECH' (LP), the National Natural Science Foundation of China (31871003, 31901053) (YH), and Ecole Doctorale 62 Sciences de la vie et de la santé, Aix-Marseille Université (BL). This article is based upon work from COST Action CA 17140 'Cancer Nanomedicine from the Bench to the Bedside' supported by COST (European Cooperation in Science and Technology).

\section{Author contribution}

LP coordinated the project. LD, ZL, AB and PG synthesized the agents, LD, ZL, AT, SG, AB, and PG made the characterization of the agents, EL, DM, and SP provided ITC and molecular modeling data, EM and PG prepared the SOJ-6 animal model, LD, YJ, and JL prepared the LIPC animal model, PG, BL, LB, and SF performed imaging experiments, MZ, WS, and YH performed toxicity study, LD, ZL, AT, PG, BL, AB, BG, YH, EL, SP, and LP analyzed data; LP wrote the paper with contribution from LD, ZL, AT, EL, SP, PG, BG, and YH. All authors proofed the manuscript.

\section{References:}

[1] M.L. James, S.S. Gambhir, Physiol. Rev. 2012, 92, 897.

[2] C. Li, Nat. Mater. 2014, 13, 110.

[3] H. Chen, W. Zhang, G. Zhu, J. Xie, X. Chen, Nat. Rev. Mater. 2017, 2, 17024.

[4] E.K.-H. Chow, D. Ho, Sci. Transl. Med. 2013, 5, 216rv214. (Ling DING: replace this by 2020

[5] E.-K. Lim, T. Kim, S. Paik, S. Haam, Y.-M. Huh, K. Lee, Chem. Rev. 2015, 115, 327.

[6] H. Maeda, J. Wu, T. Sawa, Y. Matsumura, K. Hori, J. Control. Release 2000, 65, 271. 
[7] M.A. Mintzer, M.W. Grinstaff, Chem. Soc. Rev. 2011, 40, 173.

[8] A.R. Menjoge, R.M. Kannan, D.A. Tomalia, Drug Discov. Today 2010, 15, 171.

[9] D. Ling, L. Zhenbin, D. Dinesh, K. Chai-Lin, B. Monique, P. Ling, Sci. China Mater. 2018, $61,1420$.

[10] Z. Qiao, X. Shi, Prog. Polym. Sci. 2015, 44, 1.

[11] L. Zhao, X. Shi, J. Zhao, Drug Deliv. 2017, 24, 81.

[12] L. Zhao, X. Shi, J. Zhao, in Nanotechnology Characterization Tools for Biosensing and Medical Diagnosis, (Eds: C. Kumar) Springer, Berlin, Heidelberg 2018, 509.

[13] P. Garrigue, J. Tang, L. Ding, A. Bouhlel, A. Tintaru, E. Laurini, Y. Huang, Z. Lyu, M. Zhang, S. Fernandez, L. Balasse, W. Lan, E. Mas, D. Marson, Y. Weng, X. Liu, S. Giorgio, J. Iovanna, S. Pricl, B. Guillet, L. Peng, Proc. Natl. Acad. Sci. USA 2018, 115, 11454.

[14] L. Ding, Z. Lyu, A. Tintaru, E. Laurini, D. Marson, B. Louis, A. Bouhlel, L. Balasse, S. Fernandez, P. Garrigue, E. Mas, S. Giorgio, S. Pricl, B. Guillet, L. Peng, Chem. Commun. 2020, $56,301$.

[15] D. Ni, E.B. Ehlerding, W. Cai, Angew. Chem. Int. Ed. Engl. 2019, 58, 2570.

[16] T.J. Wadas, E.H. Wong, G.R. Weisman, C.J. Anderson, Chem. Rev. 2010, 110, 2858.

[17] O. Israel, O. Pellet, L. Biassoni, D. De Palma, E. Estrada-Lobato, G. Gnanasegaran, T. Kuwert, C. la Fougère, G. Mariani, S. Massalha, D. Paez, F. Giammarile, Eur. J. Nucl. Med. Mol. Imaging 2019, 46, 1-23.

[18] R. van der Meel, E. Sulheim, Y. Shi, F. Kiessling, W.J.M. Mulder, T. Lammers, Nat. Nanotechnol. 2019, 14, 1007.

[19] Y. Tsvetkova, N. Beztsinna, M. Baues, D. Klein, A. Rix, S.K. Golombek, W.e. Al Rawashdeh, F. Gremse, M. Barz, K. Koynov, S. Banala, W. Lederle, T. Lammers, F. Kiessling, Nano Lett. 2017, 17, 4665. 
[20] B. Mitran, Z. Varasteh, R.K. Selvaraju, G. Lindeberg, J. Sörensen, M. Larhed, V. Tolmachev, U. Rosenström, A. Orlova, Int. J. Oncol. 2016, 48, 2124.

[21] T.I. Kostelnik, C. Orvig, Chem. Rev. 2019, 119, 902.

[22] E.W. Price, C. Orvig, Chem. Soc. Rev. 2014, 43, 260.

[23] We were unable to obtain well-resolved NMR spectra for In-2 because of the highly quadrupolar effect of the ${ }^{115}$ In nucleus (C. Brevard and P. Granger, Handbook of High Resolution Multinuclear NMR, John Wiley and Sons, Inc., 1981).

[24] J. Strand, H. Honarvar, A. Perols, A. Orlova, R.K. Selvaraju, A.E. Karlström, V. Tolmachev, PLoS One 2013, 8, e70028.

[25] S. Lamichhane, S. Lee, Arch. Pharmacal Res. 2020, 43, 118. 
Table of Contents:

Surface charge of supramolecular nanosystems for in vivo biodistribution: a microSPECT/CT imaging study

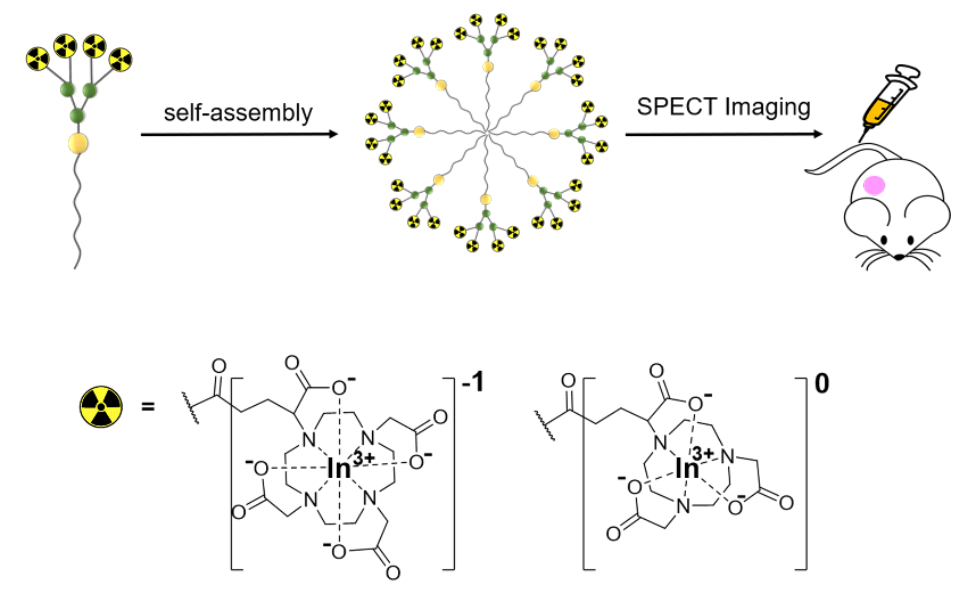

Replacing the DOTA cage with the NOTA scaffold to chelate the radionuclide $\mathrm{In}^{3+}$, the corresponding dendrimer nanosystem completely reversed the zeta-potential from negative to positive, generated a highly favorable biodistribution profile with a drastically reduced uptake in liver, and exhibited significantly improved tumor imaging. 